\title{
Response rate is not an effective mediator of learned stimulus equivalence in pigeons
}

\author{
ANDREA J. FRANK and EDWARD A. WASSERMAN \\ University of Iowa, Iowa City, Iowa
}

\begin{abstract}
We explored response rate as a possible mediator of learned stimulus equivalence. Five pigeons were trained to discriminate four clip art pictures presented during a 10-sec discrete-trial fixed interval (FI) schedule: two paired with a one-pellet reinforcer, which supported a low rate of responding, and two paired with a nine-pellet reinforcer, which supported a high rate of responding. After subjects associated one stimulus from each of these pairs with a discriminative choice response, researchers presented two new clip art stimuli during a 10-sec FI: one trained with a differential reinforcement of low rate schedule (DRL) after the FI and the other trained with a differential reinforcement of high rate schedule (DRH) after the FI. Each of the stimuli that were withheld during choice training was later shown to see if the choice responses would transfer to these stimuli. The results suggest that response rate alone does not mediate learned stimulus equivalence.
\end{abstract}

An organism's survival often depends on its effectively reacting to novel or multiple environmental stimuli. Recognizing an object at new vantage points and recognizing an organism through several sensory modalities are examples of such adaptive actions. Recognizing an object at novel vantage points may result from their physical resemblance or primary stimulus generalization (Hull, 1939). Recognizing an organism through multiple modalities may result from a learning history that entails secondary or mediated stimulus generalization (Hull, 1939).

Although it has not been as intensively investigated as primary stimulus generalization, secondary stimulus generalization may be just as useful for solving many practical behavioral problems. For example, if Steve had an unpleasant visual experience with his employer, then one might also expect Steve to have an aversive reaction to the sound of his employer's voice on the telephone. The employer in person (sight) and on the telephone (sound) are two distinctly different stimuli, yet Steve might respond to either as if they are equivalent to one another. What associative mechanisms permit such learned equivalence to develop?

One approach to uncovering the associative mechanisms underlying learned stimulus equivalence has been the investigation of superordinate categorization through many-to-one mapping (e.g., Grant \& Kelly, 2001; Grant \& Spetch, 1994; Neiman \& Zentall, 2001; Urcuioli, DeMarse, \& Zentall, 1994; Urcuioli \& Lionello-DeNolf, 2001; Urcuioli, Zentall, \& DeMarse, 1995; Wasserman, DeVolder, \& Coppage, 1992; Zentall, Sherburne, \& Urcuioli, 1993, 1995). In one type of many-to-one mapping

Correspondence concerning this article should be addressed to A. J. Frank, University of Iowa, Department of Psychology, E11 Seasore Hall, Iowa City, IA 52242 (e-mail: andrea-frank@uiowa.edu). study, organisms are trained to make a common response after two or more different discriminative stimuli. The different discriminative stimuli that are paired with a common response may form a learned equivalence class, in which these stimuli become interchangeable with one another. To test for the development of such learned equivalence, one stimulus that had been paired with a common response is associated with a new response or function. Transfer of that new response or function to the remaining stimuli is then tested. This testing often results in strong transfer to the untrained stimuli, documenting the formation of a learned equivalence class comprising distinct stimuli that had been associated with a common response.

The formation of learned equivalence classes might result from an acquired equivalence of cues: a reduction in the organism's ability to discriminate between stimuli associated with a common response (Honey \& Hall, 1989; Kaiser, Sherburne, Steirn, \& Zentall, 1997). Mediation by response or reinforcer expectation may also develop during training and later serve as a cue for the transfer of newly trained responses to untrained stimuli (e.g., Edwards, Jagielo, Zentall, \& Hogan, 1982; Grant \& Spetch, 1994; Urcuioli, 1996; Urcuioli \& DeMarse, 1994; Urcuioli et al., 1994). Acquired equivalence of cues and mediated stimulus generalization are two means by which organisms may respond appropriately in testing without explicit discrimination training.

Research by Astley and Wasserman (1999) and by Astley, Peissig, and Wasserman (2001) used many-to-one training with common reinforcing outcomes to study the formation of learned equivalence classes and the transfer of function based on a common delay to reinforcement, a common probability of reinforcement, or a common quantity of reinforcement.

Experiment 1 of Astley and Wasserman (1999) found that pigeons responded at a high rate to two classes of 
stimuli that were associated with a 1-sec delay to reinforcement, whereas pigeons responded at a low rate to two classes of stimuli that were associated with a 15-sec delay to reinforcement. When a new discriminative response was trained to one of the two classes of stimuli associated with the 1-sec delay, that new discriminative response transferred to the other class with little decrement. Likewise, when a new discriminative response was trained to one of the two classes of stimuli associated with the 15-sec delay, that new discriminative response transferred to the other class with little decrement. These learned stimulus equivalence effects also held when, in Experiment 2, the two equivalence classes were established with two different probabilities of reinforcement: .1 and .9.

In a third experiment (Astley \& Wasserman, 1999, Experiment 3), pigeons were shown four classes of stimuli, followed by different combinations of reinforcement probability and delay. Two classes were associated with certain (probability $=1.0$ ), but delayed $($ delays $=1 \mathrm{sec}$ and $15 \mathrm{sec}$ ) reinforcement, whereas two other classes were associated with immediate (delay $=0 \mathrm{sec}$ ), but uncertain (probabilities $=.9$ and .1 ) reinforcement. Despite the fact that each of the four reinforcement schedules differed from one another in the combination of probability and delay, two equivalence classes were nevertheless formed: one involving 1.0 probability with 1 -sec delay and .9 probability with 0 -sec delay, and the other involving 1.0 probability with $15-\mathrm{sec}$ delay and .1 probability with 0 -sec delay. Critically, the former two schedules involved higher rates of reinforcement than the latter two schedules, suggesting that reinforcement or hedonic value served as the psychological "glue" for learned stimulus equivalence.

Relatedly, Astley et al.'s (2001) Experiment 1 explored the role of the number of food pellets (one vs. five) in learned stimulus equivalence. Two classes of stimuli (C1 and C2) were associated with a one-pellet reinforcer, whereas two other classes of stimuli (C3 and C4) were associated with a five-pellet reinforcer. The pigeons responded similarly with a low rate of pecking in the presence of the stimuli associated with the one-pellet reinforcer ( $\mathrm{C} 1$ and $\mathrm{C} 2$ ), and they responded similarly with a high rate of pecking in the presence of the stimuli associated with a five-pellet reinforcer (C3 and C4). One class of stimuli from each group (C1 and $\mathrm{C} 3$ ) was then associated with new responses: Pecking a blue key was reinforced in the presence of the C1 stimuli, whereas pecking a green key was reinforced in the presence of the C3 stimuli. During testing, pigeons' responses were consistent with a common reinforcer quantity: Pigeons pecked the blue key in the presence of the $\mathrm{C} 2$ stimuli, whereas they pecked the green key in the presence of the $\mathrm{C} 4$ stimuli. In other words, the pigeons had formed learned equivalence classes that were based on a common reinforcer of one pellet ( $\mathrm{C} 1$ and $\mathrm{C} 2)$ or five pellets (C3 and $\mathrm{C} 4)$.

In both Astley and Wasserman (1999) and Astley et al. (2001), the hedonic value of the reinforcement schedule appeared to be the common factor that linked the distinct sets of stimuli, but the different rates of response that were produced by the different hedonic outcomes could also have mediated choice responding. Earlier, Urcuioli (1984) had reported that the different response patterns arising on DRL 3-sec versus FR 10 reinforcement schedules can function as mediating cues in a matching-tosample paradigm.

Astley and colleagues statistically assessed whether differences in response rate to the discriminative stimuli were associated with differences in choice responding to the training and testing stimuli. Response rate did not appear to have been a key mediator of choice responding because correlational analyses failed to find the predicted positive correlation between differences in response rate to the discriminative stimuli and differential choice responding.

The purpose of the present investigation was to experimentally determine whether the rate of responding alone might be the effective factor that mediates learned stimulus equivalence, regardless of how that common response rate is generated. In other words, can two stimuli become interchangeable with one another when one stimulus produces a high (or low) rate of responding because it is followed by a large (or small) quantity of reinforcement and the other stimulus produces a high (or low) rate of responding because of a direct schedule requirement (i.e., two qualitatively different methods of response rate generation such as different numbers of food pellets vs. DRL and DRH schedules of reinforcement)?

We looked into this possibility by first associating two stimuli (A and B) with a one-pellet reinforcer and two stimuli ( $C$ and $D)$ with a nine-pellet reinforcer. One stimulus from each of these two conditions (A and C) was later associated with one of two different choice responses followed by random reinforcers of one or nine pellets. Transfer of the choice responses to the two remaining stimuli (B and D) was then tested. The pigeons responded to the $\mathrm{B}$ and D stimuli at a very high level of accuracy-learned stimulus equivalence. Once the formation of the two learned equivalence classes $(\mathrm{A}+\mathrm{B}$ and $\mathrm{C}+\mathrm{D})$ had been clearly established, two new stimuli ( $\mathrm{E}$ and $\mathrm{F}$ ) were trained to elicit different rates of responding via differential reinforcement of low rate (DRL) or differential reinforcement of high rate (DRH) schedules. A random one- or nine-pellet reinforcer followed proper performance on the DRL or DRH procedures. Finally, the DRL and DRH stimuli were tested for transfer of the choice responses that had previously been trained to the A and $\mathrm{C}$ stimuli. The results strongly suggested that response rate alone was unlikely to have mediated learned stimulus equivalence in this experiment.

\section{METHOD}

\section{Participants}

Five feral pigeons (Columba livia) successfully completed all phases of the experiment. ${ }^{1}$ The birds were maintained at $85 \%$ of their free-feed weights on a 14:10-h light:dark schedule with free access to water and grit. The pigeons had already been trained to peck the center button of the apparatus (see procedures) in unrelated experiments. 


\section{Apparatus}

Four custom-built operant chambers were used for training and testing (Young \& Wasserman, 1997). Centered on the front wall of each chamber was a $7 \times 7 \mathrm{~cm}$ square opening with four $1.9-\mathrm{cm}$ diameter circular openings $2.3 \mathrm{~cm}$ from each corner. Behind the openings was a clear glass touch screen (Accutouch Model No. 002744-FTM-KI, Elographics, Oak Ridge, TN). Reinforcers were 45-mg pellets (Pigeon Pellets Formula C1, Research Diets, New Brunswick, NJ). Computer programs were created with Hypercard (Version 2.4).

\section{Stimuli}

The stimuli were six color clip art images - butterfly, snail, plant, blue flower, pink flower, and beetle-labeled A-F, respectively. Stimuli B, D, E, and F were counterbalanced across birds; however, Stimulus A (butterfly) and Stimulus C (pink flower) were the same for all birds because these two stimuli served as the choice training stimuli throughout the entire experiment.

\section{Procedure}

Pellet training. Our pellet training and choice training methods were based on procedures used in Astley et al.'s (2001) Experiment 1. Pellet training involved the random presentation of four distinct picture stimuli in the center of the screen (Stimuli A, B, C, and D) for a 10 -sec fixed interval (FI) followed by either a one- or a nine-pellet reinforcer and a variable 5- to 10 -sec intertrial interval (ITI). Stimuli A and B were followed by one pellet, whereas Stimuli $\mathrm{C}$ and $\mathrm{D}$ were followed by nine pellets. There were 12 presentations of each stimulus, for 48 total trials per daily session. Training lasted 32 days. We required that the pigeons strongly discriminate the one- and nine-pellet stimuli in order to progress to choice training (a difference of 0.40 pecks per sec between the one- and nine-pellet stimuli served as the cutoff for continuing in the study); without a strong separation in response rate, any mediation of learned stimulus equivalence by different rates of responding would have been most unlikely.

Choice training. Choice training trials were conducted using only the $\mathrm{A}$ and $\mathrm{C}$ stimuli accompanied by the appearance of the two choice buttons. Trials began with a 10 -sec FI followed by presentation of the choice buttons at the bottom left and right corners of the picture stimulus. Selection of the blue choice button (bottom left) in the presence of Stimulus A was reinforced, whereas selection of the green choice button (bottom right) in the presence of Stimulus $\mathrm{C}$ was reinforced. A correct choice was randomly followed by one or nine pellets; random numbers of pellets were given on these choice training trials so that the pigeons could not selectively associate these choice responses with either quantity of reinforcement. An incorrect choice resulted in a variable 5- to 10-sec intertrial interval (ITI), during which the houselight was dark, followed by repetition of the same trial until the bird chose the correct color button.

Choice training sessions contained blocks of stimuli that alternated twice: 3 of each of the four pellet training stimuli (12 pellet training trials) and 6 of each of the two choice training stimuli (12 choice training trials). Sessions contained 48 total trials and continued until each bird reached a criterion of $90 \%$ or better on the choice training trials for 2 consecutive days.

Transfer test. Transfer test trials were composed of the B and D stimuli that were presented during a 10 -sec FI, followed by presentation of the blue and green choice color buttons at the bottom left and right corners of the picture stimulus. Choice of either color button randomly resulted in a one- or nine-pellet reinforcer. If the birds had learned to treat Stimulus A and Stimulus B as functionally equivalent to one another because of their association with a common one-pellet reinforcer, then one would expect the birds to choose the blue choice button in the presence of Stimulus B. Similarly, if the birds had learned to treat Stimulus C and Stimulus D as functionally equivalent to one another because of their association with a common nine-pellet reinforcer, then one would expect the birds to choose the green choice button in the presence of Stimulus D. Either a one- or nine-pellet reinforcer randomly followed choice of either color button on transfer test trials.

In this phase, stimulus presentation was blocked. The first block consisted of 8 pellet training trials (two trials of each of the four picture stimuli: A, B, C, and D). The second block contained 12 choice training trials ( 6 trials of each of the two choice training stimuli: A and $\mathrm{C}$ ). The third block consisted of 16 choice training trials (A and $\mathrm{C}$ ) randomly mixed with 8 transfer test trials (B and D). Transfer test sessions contained 44 total trials and were given on 8 consecutive days. Mean choice accuracy across these 8 days had to result in at least $75 \%$ correct on transfer test trials (B and D) for the pigeon to continue in the experiment; only birds meeting this stringent $75 \%$ criterion were retained in order to maintain a high discrimination baseline from which we could sensitively measure any drops in performance. Pigeons were removed from the experiment immediately after failing to meet the $75 \%$ criterion. We reasoned that, if pigeons could not maintain a high discrimination on the transfer test, then they would not show a high discrimination on the final test (see below).

DRL-DRH training. Differential reinforcement of low (DRL) and high (DRH) rate responding was arranged on these training trials. On the DRL trials, the birds were presented with a new picture stimulus, E, for $10 \mathrm{sec}$, followed by the DRL schedule. During the DRL schedule, the stimulus remained on the screen, and the bird had to peck the stimulus twice, at least $3 \mathrm{sec}$ apart. On DRH trials, following $10 \mathrm{sec}$ with a new stimulus, F, the bird had to peck twice within $3 \mathrm{sec}$. On both types of trials, if the bird did not wait the correct amount of time (DRL) or respond within the correct amount of time (DRH), then the clock was reset and the DRL or DRH schedule was repeated until the bird responded appropriately. Random reinforcers of one or nine pellets followed the completion of the DRL or DRH requirement, so that neither the $\mathrm{E}$ nor the $\mathrm{F}$ stimulus was selectively associated with a particular reinforcer quantity.

DRL-DRH training sessions consisted of 24 trials of each of the two stimuli, for 48 total trials. DRL-DRH training sessions were alternated every other day with choice training sessions to ensure that the birds did not forget how to respond properly on the pellet training trials or on the choice training trials. DRL-DRH training lasted for 8 days; this period was long enough to see a very strong separation in peck rates to the DRL and the DRH stimuli.

Final test. The final test trials consisted of showing the $\mathrm{E}$ and $\mathrm{F}$ stimuli on a $10-\mathrm{sec}$ FI schedule, followed by presentation of the blue and green color choice buttons at the bottom left and right corners of the picture stimuli. One or nine pellets randomly followed choice of either button, so that the E and F stimuli were not selectively associated with either quantity of reinforcement.

Final test sessions comprised blocked trials similar to the transfer test sessions. The first block contained 2 training trials of each stimulus from pellet training and DRL-DRH training sessions (A, B, C, D, E, and F) for 12 total trials. The second block contained 12 choice training trials (A and C: 6 of each stimulus). The third block contained 16 choice training trials (A and C: 8 of each stimulus) randomly mixed with 8 transfer test trials (B and D: 4 of each stimulus) and 8 final test trials (E and F: four of each stimulus). Final test sessions contained 56 total trials and were presented every 3rd day after DRL-DRH training and choice training sessions. Eight alternations of DRL-DRH training, choice training, and final test sessions were conducted.

\section{RESULTS}

\section{Pellet Training}

Mean peck rates to the stimuli associated with nine pellets (C and D) were very similar to each other $(2.00$ mean pecks per sec and 1.95 mean pecks per sec, respectively), and mean peck rates to the stimuli associated with 
one pellet (A and $B$ ) were also very similar to each other ( 0.79 mean pecks per sec and 0.99 mean pecks per sec, respectively). So, mean peck rates to the nine-pellet stimuli were averaged together and mean peck rates to the one-pellet stimuli were also averaged together. During the 32 days of pellet training, all 5 pigeons responded faster to the two stimuli associated with nine pellets $(\mathrm{C}$ and $\mathrm{D}$; mean pecks per sec $=$ 1.98) than to the two stimuli associated with one pellet (A and $\mathrm{B}$; mean pecks per $\mathrm{sec}=0.89)$. A full-factorial stimulus (one or nine pellets) $\times$ training $(32$ days $) \times$ bird (random effect) analysis of variance (ANOVA) of response rate yielded only a significant stimulus main effect $[F(1,4)=$ $\left.34.84, p<.004, M S_{\text {Den }}=2.72\right]$. The rejection criterion of .05 was used for all analyses. Mean response rates to the two 1-pellet (A and B) stimuli and to the two nine-pellet stimuli (C and D) were analyzed because there should be no difference in the response rates to the two stimuli associated with a common reinforcer. This averaging was done for the response rate analyses in all phases unless otherwise stated.

The reliable difference in response rates clearly showed that the birds very effectively learned the different stimulusreinforcer associations. In fact, there was a 0.50 peck per sec difference between the one- and nine-pellet stimuli by the 3rd day of training. It is possible that there could have been a greater separation between the high and low peck rates if more disparate reinforcers had been used (e.g., food vs. no food).

\section{Choice Training}

The mean number of days that it took the 5 birds to meet the criterion of $90 \%$ correct choice to Stimuli A and $\mathrm{C}$ for 2 consecutive days was 4.60 days. Three birds took 4 days, 1 took 5 days, and the other took 6 days.

Throughout choice training, all 5 pigeons responded faster to the $\mathrm{C}$ stimulus (associated with nine pellets) during warm-up trials (mean pecks per sec $=1.95$ ) and during choice trials (mean pecks per sec $=1.80$ ) than they did to the A stimulus (associated with one pellet) during warm-up trials (mean pecks per sec $=1.05$ ) and during choice trials (mean pecks per $\mathrm{sec}=1.08$ ). One-way ANOVAs showed no significant difference in response rate to Stimulus $C$ during warm-up or choice trials $[F(1,44)=0.61 ; p<$ $\left..44 ; M S_{\text {Den }}=0.39\right]$ and no significant difference in response rate to Stimulus A during warm-up or choice trials $\left[F(1,44)=0.05 ; p<.82 ; M S_{\text {Den }}=0.33\right]$. Thus, the rate of responding on warm-up and choice trials was similar, allowing response rate to guide choice responses if peck rate was the mediating factor.

\section{Transfer Test}

Throughout the 8 days of transfer testing, all 5 pigeons responded faster to Stimulus $C$ (mean peck rate $=2.29$ ) and Stimulus D (mean peck rate $=2.47$ ) than to Stimulus A $($ mean peck rate $=1.33)$ and Stimulus B (mean peck rate $=1.21)$. All 5 pigeons responded faster to the stimuli associated with nine pellets $(\mathrm{C}$ and $\mathrm{D}$; mean peck rate $=$ 2.38) than to the stimuli associated with one pellet (A and $\mathrm{B}$; mean peck rate $=1.27$ ) during warm-up trials. A full-factorial stimulus (one and nine pellets) $\times$ test day $(8$ days) $\times$ bird (random effect) ANOVA of response rate yielded only a significant stimulus main effect $[F(1,4)=$ $\left.13.51, p<.0213, M S_{\text {Den }}=7.31\right]$.

Throughout the 8 days of transfer testing, mean accuracy on choice trials (A and $\mathrm{C}$ ) was $92.66 \%$ correct, and mean accuracy on transfer test trials (B and D) was $88.44 \%$ correct. Mean accuracy on choice trials was significantly above chance $[Z=21.54, N=640]$ as was mean accuracy on transfer test trials $[Z=13.69, N=320]$. In addition, a full-factorial stimulus (choice and transfer) $\times$ test day $(8$ days) ANOVA yielded no significant differences. Mean accuracy to Stimuli A and C combined (choice) and to Stimuli B and D combined (transfer) were again analyzed because of the common choice training of Stimuli A and $\mathrm{C}$ and the common choice training of Stimuli B and D.

We also analyzed the choice accuracy of all 10 pigeons that progressed beyond pellet training: the 5 birds that continued in the experiment and the 5 birds that were removed because their accuracy on the transfer test trials fell below 75\% correct during transfer testing. We conducted this analysis to see if the collective performance of all 10 pigeons was similar to the results of Astley et al. (2001), who did not exclude birds from their study based on a choice criterion. For the 8 days of transfer testing, the mean accuracy of all 10 pigeons on the choice trials was significantly above chance at $91.25 \%$ correct $(Z=29.54$, $N=1280$ ), as was the mean accuracy of all 10 pigeons on the transfer test trials at $79.53 \%$ correct $(Z=14.98, N=$ 640). On transfer test trials, the overall accuracy of all 10 pigeons here $(79.53 \%)$ was numerically higher than the overall accuracy $(75.26 \%)$ of the pigeons in Astley et al. (2001; Experiment 1).

\section{DRL-DRH Training}

Throughout the 8 days of DRL-DRH training, all 5 pigeons responded faster to the stimulus associated with the DRH schedule $(\mathrm{F}$; mean peck rate $=1.99)$ than to the stimulus associated with the DRL schedule (E; mean peck rate $=0.49$ ). The pigeons learned the DRL-DRH discrimination very quickly. Across the 5 birds' training performance, there was a mean difference of 0.93 pecks per sec on the first day of training. Response rates on the DRH schedule were relatively stable during training, whereas response rates on the DRL schedule declined during training. A full-factorial stimulus $(\mathrm{E}$ and $\mathrm{F}) \times$ training day (8 days) $\times$ bird (random effect) ANOVA yielded significant stimulus $\left[F(1,4)=38.63, p<.003, M S_{\text {Den }}=1.17\right]$ and training day $\left[F(1,60)=13.98, p<.0004, M S_{\text {Den }}=\right.$ $0.02]$ main effects. The stimulus $\times$ training day interaction was not significant $\left[F(1,4)=4.09, p<.113, M S_{\text {Den }}=\right.$ $0.14]$.

This reliable difference in response rate to Stimulus E and Stimulus F shows that the birds clearly learned the stimulus-response associations in DRL-DRH training.

\section{Final Test}

Mean response rates on the warm-up training trials (one pellet, nine pellets, DRL, and DRH) across the 8 days of 
final testing are shown in Figure 1 (top). Pigeons maintained a much higher rate of responding to the two stimuli associated with nine pellets $(\mathrm{C}$ and $\mathrm{D}$; mean peck rate $=$ 2.31) than to the two stimuli associated with one pellet (A and $\mathrm{B}$; mean peck rate $=0.99$ ). Response rate was also much higher to the stimulus associated with the DRH schedule $(\mathrm{F}$; mean peck rate $=2.07)$ than to the stimulus associated with the DRL schedule $(\mathrm{E}$; mean peck rate $=$ 0.44). A full-factorial stimulus (one pellet, nine pellets, DRL, DRH) $\times$ test day $(8$ days $) \times$ bird (random effect) ANOVA of response rates to the stimuli associated with one pellet (A and B), nine pellets (C and D), DRL (E), and DRH (F) yielded only a significant stimulus main effect
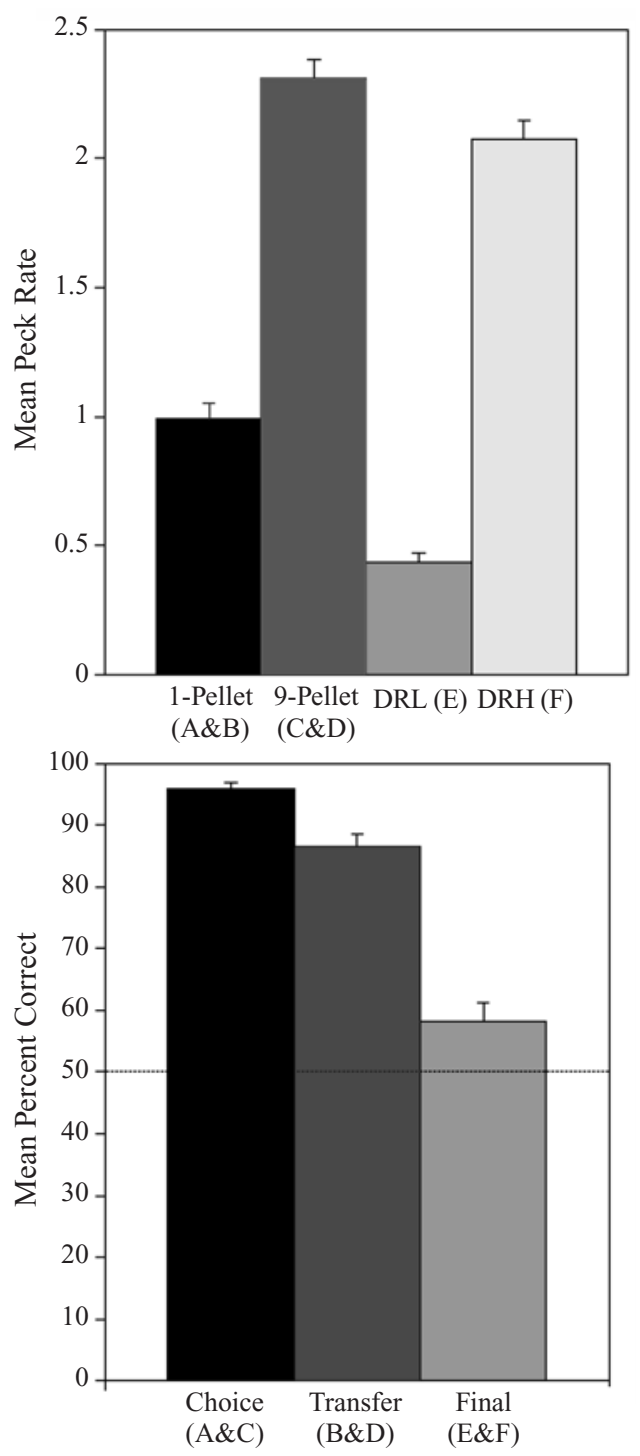

Figure 1. (Top) Mean peck rates during warm-up trials to the stimuli associated with one pellet (Stimuli $\mathrm{A}$ and B), nine pellets (Stimuli C and D), DRL (Stimulus E), and DRH (Stimulus F) across the 8 days of final testing. (Bottom) Mean accuracy during testing on the choice, transfer, and final test trials across the 8 days of final testing.
$\left[F(3,12)=36.68, p<.0001, M S_{\text {Den }}=2.39\right]$. Follow-up tests yielded a significant difference between the onepellet $(\mathrm{A}$ and $\mathrm{B})$ and nine-pellet $(\mathrm{C}$ and $\mathrm{D})$ stimuli $[t(2,12)=$ $-7.63, p<.0001]$ and between the DRL (E) and DRH (F) stimuli $[t(2,12)=-6.69, p<.0001]$. Response rate to the DRH (F) stimulus was similar to the response rate to the nine-pellet stimuli ( $\mathrm{C}$ and $\mathrm{D})$; response rate to the DRL (E) stimulus was even lower than response rate to the one-pellet stimuli (A and B). There was no significant difference between response rates to the nine-pellet stimuli and the DRH stimulus $[t(2,12)=1.12, p<.287]$; there was a significant difference between response rates to the one-pellet stimuli and the DRL stimulus $[t(2,12)=2.61$; $p<.023]$.

Mean accuracies on choice trials (A and C), transfer test trials (B and D), and final test trials ( $\mathrm{E}$ and F; DRL and DRH schedules, respectively) are shown in Figure 1 (bottom). Mean accuracy on choice trials was $95.78 \%$, on transfer test trials, $86.56 \%$; and on final test trials, $58.13 \%$; all scores were significantly above chance $[Z=23.20$, $N=640 ; Z=13.03, N=320$; and $Z=2.85, N=320$, respectively]. A full-factorial stimulus (choice, transfer, and final test stimuli) $\times$ test day $(8$ days $) \times$ bird (random effect) ANOVA yielded only a significant stimulus main effect $\left[F(2,8)=19.49, p \leq .0008, \mathrm{MS}_{\text {Den }}=0.78\right]$. There was no significant difference in accuracy between choice trials and transfer test trials in follow-up tests $[t(1,8)=$ $1.52, p<.167]$; there were significant differences in accuracy between choice trials and final test trials $[t(1,8)=$ $6.22, p<.0003$ ] and between transfer test trials and final test trials $[t(1,8)=4.07, p<.004]$.

\section{DISCUSSION}

The goal of our experiment was to see if different rates of responding could effectively mediate learned equivalence class formation, even when those disparate response rates were produced by qualitatively different experimental methods: different numbers of food pellets on the same reinforcement schedule or the same number of food pellets on different reinforcement schedules. Astley and Wasserman (1999) and Astley et al. (2001) reported that learned stimulus equivalence was produced by different hedonic outcomes, such as different probabilities of reinforcement, different delays to reinforcement, and different quantities of reinforcement. Importantly, different rates of responding to the discriminative stimuli were generated by all of these disparate outcomes, raising the possibility that response rate itself - and not some factor that generated that rate-was the critical factor in creating learned stimulus equivalence.

In the present experiment, it might have been expected that learned equivalence classes arose because of the low and generally similar rates of responding among the A, $\mathrm{B}$, and $\mathrm{E}$ stimuli and because of the high and generally similar rates of responding among the $\mathrm{C}, \mathrm{D}$, and $\mathrm{F}$ stimuli (Figure 1, top). Mean accuracy on choice trials (A and $\mathrm{C})$, transfer test trials (B and D), and final test trials (E 
and F) was significantly above chance in all three cases (Figure 1, bottom).

If peck rate effectively mediated learned stimulus equivalence, then differences in the rates of responding and the levels of choice accuracy to the three pairs of stimuli should have been positively correlated. For example, the largest difference in response rates to the warm-up training stimuli was between the final test stimuli (DRL $[\mathrm{E}]$ and DRH [F]; mean peck rate difference $=1.64$ ). The next largest difference in response rates was between the transfer stimuli (B and D; mean peck rate difference $=$ 1.54). The smallest difference in response rates was between the choice stimuli (A and C; mean peck rate difference $=1.09$ ). (The difference between $\mathrm{A}$ and $\mathrm{C}$ was smaller than between $\mathrm{B}$ and $\mathrm{D}$, because $\mathrm{A}$ and $\mathrm{C}$ were randomly followed by one- or nine-pellet reinforcers on choice training trials.) If response rate effectively mediated learned stimulus equivalence, then one would expect mean choice accuracy to have been ranked as follows: final test stimuli $(\mathrm{E}$ and $\mathrm{F})>$ transfer stimuli $(\mathrm{B}$ and $\mathrm{D})>$ choice stimuli (A and C). Precisely the opposite ranking was actually observed (Figure 1, bottom). Indeed, a statistically significant $(p<.01)$ correlation of -0.45 held between the difference in response rate and choice accuracy, based on individual pigeons' performance during the 8 days of final testing.

The conclusion that peck rate alone is unlikely to be the mediator of equivalence class formation in our experiment is not limited to the pigeons' relative rates of responding to the discriminative stimuli. Consideration of the pigeons' absolute rates of responding to the discriminative stimuli also fails to account for the learned equivalence classes that emerged in our experiment.

Figure 2 (bottom, all birds) shows that there was a drop in the pigeons' choice of the response associated with nine food pellets when they were shown the F(DRH) stimulus. This drop is surprising, given the high and similar rates of responding that were controlled by Stimuli C, D, and F. Also surprising was the large rise in the pigeons' choice of the response associated with nine food pellets when they were shown the E (DRL) stimulus. The pigeons pecked at even lower rates to Stimulus E than they did to Stimuli $\mathrm{A}$ and $\mathrm{B}$. This lower response rate should have prompted lower, not higher levels of pigeons' choosing the ninepellet response if the pigeons' absolute rate of responding were directly related to the probability of choosing the nine-pellet key over the one-pellet key.

Perhaps we did not match the low and high rates of response closely enough with our DRL-DRH training procedure. It is possible that using a different number of pellets during pellet training (e.g., food vs. no food) might have resulted in peck rates more similar to those resulting from the DRL-DRH training procedure. However, examination of the percentage of nine-pellet key choice and peck rate scores for the individual pigeons disclosed that the poorest discriminated stimulus in both conditions (DRL and DRH) was the final test stimulus (E or F), regardless of how similar or disparate the peck rates were for these stimuli compared with the training and transfer stimuli (Figure 2). For example, the peck rates to the onepellet stimuli (A and B) and the DRL stimulus (E) by Bird 54W (Figure 2) were ordered: B $<\mathrm{E}<\mathrm{A}$. Even though the peck rate for the $\mathrm{E}$ stimulus fell between that of the $\mathrm{A}$ and B stimuli, the percentage of nine-pellet key choices made when presented with the E stimulus was the highest of the three stimuli. In other words, Bird 54W showed the poorest discrimination when presented with the E stimulus regardless of the fact that the peck rate to this stimulus fell between that of the two one-pellet stimuli (A and B). One might have expected that the poorest discrimination would have occurred when this bird was shown the stimulus associated with the highest peck rate (Stimulus A) because this stimulus controlled a rate of response closest to Stimulus C.

Insofar as peck rate is concerned, Figure 2 shows that it does not matter in what order each trio of stimuli fell: Stimulus E (DRL) was the poorest discriminated of the three low-rate stimuli (A, B, and E) for each of the 5 birds and (with the exception of Bird 54W), Stimulus F (DRH) was the poorest discriminated of the three high-rate stimuli (C, D, and F).

Another issue arises in connection with Figure 2: Why might the pigeons have generally chosen the nine-pellet report key in the presence of both the $\mathrm{E}$ and $\mathrm{F}$ stimuli, even though one- and nine-pellets equally often followed these stimuli during training and testing? Logan (1965) suggested that "if new principles are required by conditionaloutcome choices, then a preference might appear between alternatives that are otherwise equally reinforced" (p. 469). Because the birds had never previously seen the $\mathrm{E}$ and $\mathrm{F}$ stimuli in the presence of the choice keys, this novel testing condition could have produced a preference for one choice key over the other - here, the choice key associated with the more favorable outcome (nine pellets). Another possible explanation for the pigeons' choice of the key associated with nine pellets is that the random delivery of one or nine pellets of food after stimuli $\mathrm{E}$ and $\mathrm{F}$ rendered these stimuli more like the nine pellet-paired stimuli (C and D) than the one pellet-paired stimuli (A and B) (Dinsmoor, 1983).

All of these findings and considerations lead us to suggest that our pigeons formed learned equivalence classes based on the quantity of food reinforcement. According to this account, the $\mathrm{E}$ and $\mathrm{F}$ stimuli (associated with the DRL and DRH schedules, respectively) should not have entered into learned equivalence classes either with the stimuli that were associated with one pellet (A and B) or with the stimuli that were associated with nine pellets (C and D) because the stimuli associated with the DRL (E) and the DRH (F) schedules were randomly followed by $e i-$ ther one- or nine-pellets. We therefore conclude that there is little or no empirical support for sample-specific behaviors (here, in the form of different peck rates) having served as the main discriminative stimuli for our pigeons' choice responding. Our conclusion is also consistent with the results of Zentall and Sherburne (1994), who found that differential sample responding and differential outcomes can act on behavior independently of one another. 
31B
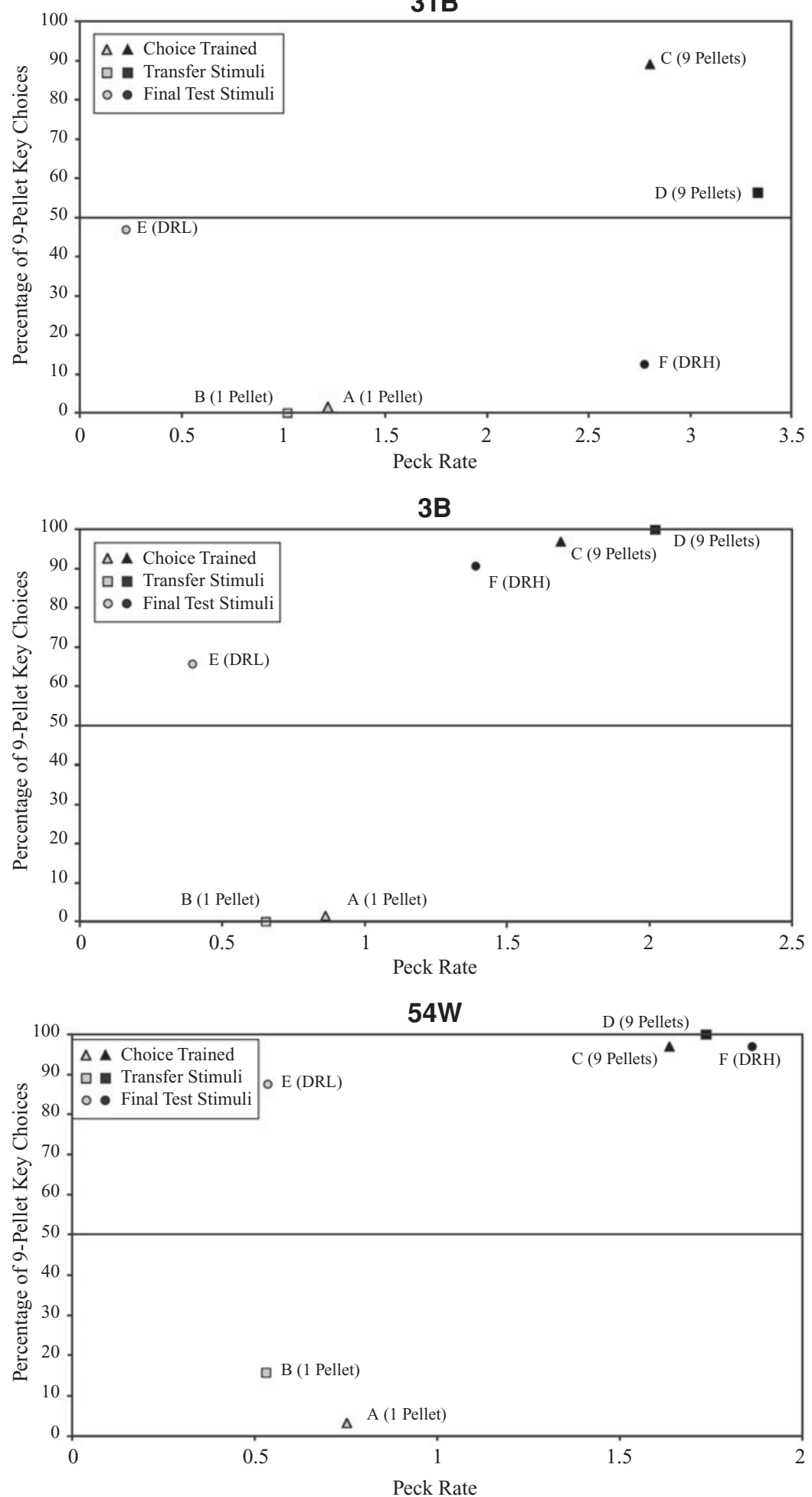

Figure 2. Scatterplots of the mean percentage choice of the key associated with the nine-pellet stimuli as a function of mean peck rate for each of the 5 birds. Also shown is a scatterplot for the average of the 5 birds. 
$58 \mathrm{~W}$

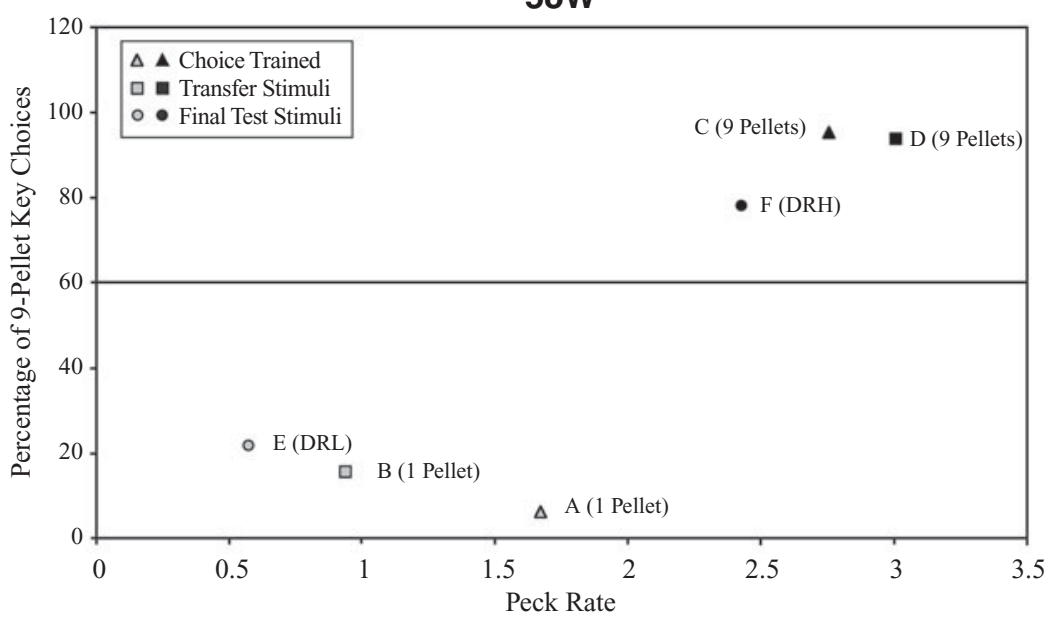

8B

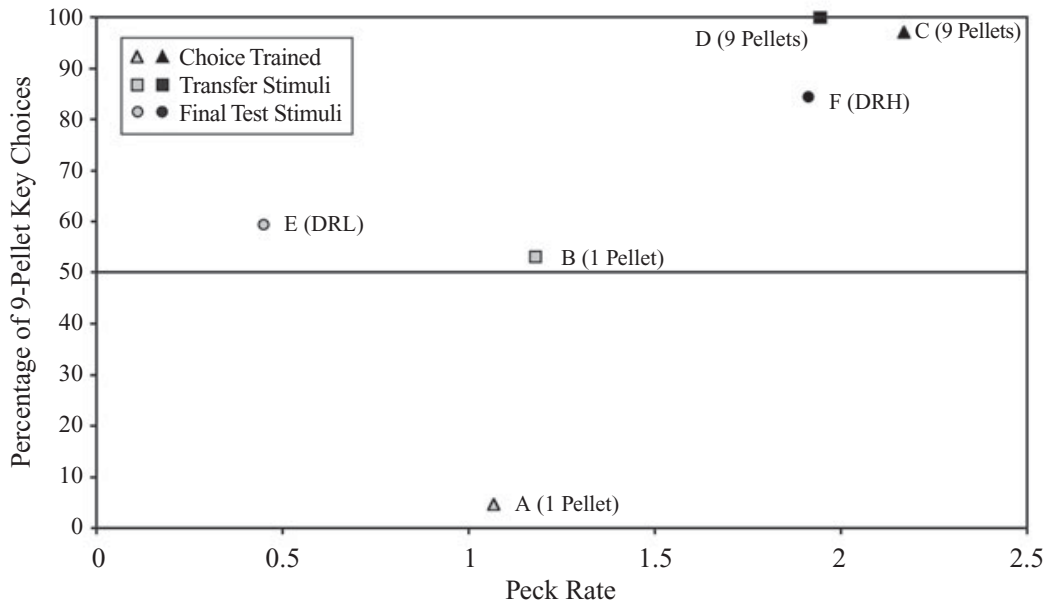

All Birds

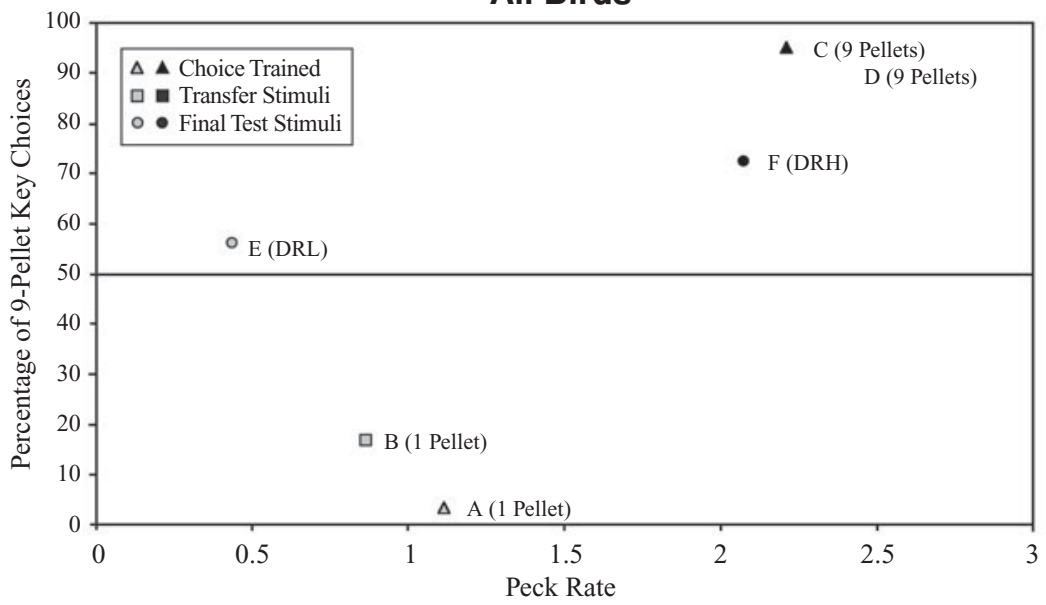

Figure 2. (Continued).

The possibility remains, however, that rate of responding may be a mediator, just not the main discriminative cue on which our pigeons based their choice responses. A small contribution by peck rate may be the reason why choice accuracy to the final test stimuli was slightly, but significantly, above chance. Urcuioli (1984) found that sample-specific behaviors can overshadow the sample as a cue for choosing a comparison stimulus in a matching 
task. The fact that we gave differing numbers of pellets for reinforcement may somehow have reduced that overshadowing effect; this might be why the E (DRL) and F (DRH) stimuli were not included in the respective learned equivalence classes with the stimuli that were followed by a similar low or high rate of responding. In addition, the possibility remains that the force or the location of the pigeons' pecks or their precise time of occurrence during the trial might have served as mediators of choice responding. These possibilities seem remote ones, but we are unable to eliminate them on the basis of the data that we have reported.

In conclusion, based on our results and those of Astley and Wasserman (1999) and Astley et al. (2001), it appears most likely that hedonic or reinforcement value mediates learned stimulus equivalence based on the number, probability, and delay of reinforcement. Although stimulusspecific behaviors may be produced by the prevailing reinforcement schedules, those behaviors do not appear to directly or strongly mediate learned stimulus equivalence.

\section{REFERENCES}

Astley, S. L., Peissig, J. J., \& Wasserman, E. A. (2001). Superordinate categorization via learned stimulus equivalence: Quantity of reinforcement, hedonic value, and the nature of the mediator. Journal of Experimental Psychology: Animal Behavior Processes, 27, 252-268.

Astley, S. L., \& Wasserman, E. A. (1999). Superordinate category formation in pigeons: Association with a common delay or probability of food reinforcement makes perceptually dissimilar stimuli functionally equivalent. Journal of Experimental Psychology: Animal Behavior Processes, 25, 415-432.

Dinsmoor, J. A. (1983). Observing and conditioned reinforcement. Behavioral \& Brain Sciences, 6, 693-728.

Edwards, C. A., Jagielo, J. A., Zentall, T. R., \& Hogan, D. E. (1982). Acquired equivalence and distinctiveness in matching to sample by pigeons: Mediation by reinforcer-specific expectancies. Journal of Experimental Psychology: Animal Behavior Processes, 8, 244-259.

Grant, D. S., \& Kelly, R. (2001). Many-to-one matching with temporal and hedonic samples in pigeons. Learning \& Motivation, 32, 477-498.

Grant, D. S., \& Spetch, M. L. (1994). Mediated transfer testing provides evidence for common coding of duration and line samples in many-to-one matching in pigeons. Animal Learning \& Behavior, 22, 84-89.

Honey, R. C., \& HaLl, G. (1989). Acquired equivalence and distinctiveness of cues. Journal of Experimental Psychology: Animal Behavior Processes, 15, 338-346.

HulL, C. L. (1939). The problem of stimulus equivalence in behavior theory. Psychological Review, 46, 9-30.

Kaiser, D. H., Sherburne, L. M., Steirn, J. N., \& Zentall, T. R. (1997). Perceptual learning in pigeons: Decreased ability to discriminate samples mapped onto the same comparison in many-to-one matching. Psychonomic Bulletin \& Review, 4, 378-381.
Logan, F. A. (1965). Conditional-outcome choice behavior in rats. Psychological Review, 69, 467-476.

Neiman, E. R., \& Zentall, T. R. (2001). Common coding of samples associated with the same comparison: The nature of the common representation. Learning \& Motivation, 32, 367-382.

Urcuioli, P. J. (1984). Overshadowing in matching-to-sample: Reduction in sample-stimulus control by differential sample behaviors. Animal Learning \& Behavior, 12, 256-264

URCUIOLI, P. J. (1996). Acquired equivalences and mediated generalization of pigeon's matching-to-sample. In T. R. Zentall \& P. M. Smeets (Eds.), Stimulus class formation in humans and animals (pp. 55-90). Amsterdam: Elsevier.

URCUIOLI, P. J., \& DeMarse, T. B. (1994). On the relationship between differential outcomes and differential sample responding in matchingto-sample. Journal of Experimental Psychology: Animal Behavior Processes, 20, 249-263.

Urcuioli, P. J., DeMarse, T. B., \& Zentall, T. R. (1994). Some properties of many-to-one matching with hue, response, and food samples: Retention and mediated transfer. Learning \& Motivation, 25, 175-200.

Urcuioli, P. J., \& Lionello-DeNolf, K. M. (2001). Some tests of the anticipatory mediated generalization model of acquired sample equivalence in pigeons' many-to-one matching. Animal Learning \& Behavior, 29, 265-280.

Urcuioli, P. J., Zentall, T. R., \& DeMarse, T. B. (1995). Transfer to derived sample-comparison relations by pigeons following many-toone versus one-to-many matching with identical training relations. Quarterly Journal of Experimental Psychology, 48B, 158-178.

Wasserman, E. A., DeVolder, C. L., \& Coppage, D. J. (1992). Nonsimilarity-based conceptualization in pigeons via secondary or mediated generalization. Psychological Science, 3, 374-379.

YounG, M. E., \& Wasserman, E. A. (1997). Entropy detection by pigeons: Response to mixed visual displays after same-different discrimination training. Journal of Experimental Psychology: Animal Behavior Processes, 23, 157-170.

Zentall, T. R., \& Sherburne, L. M. (1994). Role of differential sample responding in the differential outcomes effect involving delayed matching by pigeons. Journal of Experimental Psychology: Animal Behavior Processes, 20, 390-401.

Zentall, T. R., Sherburne, L. M., \& Urcuioli, P. J. (1993). Common coding by pigeons in a many-to-one delayed matching task as evidenced by facilitation and interference effects. Animal Learning \& Behavior, 21, 233-237.

Zentall, T. R., Sherburne, L. M., \& Urcuioli, P. J. (1995). Coding of hedonic and nonhedonic samples by pigeons in many-to-one delayed matching. Animal Learning \& Behavior, 23, 189-196.

\section{NOTE}

1. Thirteen pigeons were actually trained in the experiment. Three birds were removed after the pellet training phase because their peck rates to the one-pellet and nine-pellet stimuli did not differ by at least 0.40 pecks per sec. Five birds were removed because their discriminative performance on the transfer test fell below $75 \%$ correct to the testing stimuli. See text for further details, discussion, and data.

(Manuscript submitted February 9, 2004; revision accepted for publication October 5, 2004.) 